Bull. Korean Math. Soc. 46 (2009), No. 6, pp. 1229-1236

DOI 10.4134/BKMS.2009.46.6.1229

\title{
QUASI-INNER FUNCTIONS OF A GENERALIZED BEURLING'S THEOREM
}

\author{
Yun-Su KIM
}

\begin{abstract}
We introduce two kinds of quasi-inner functions. Since every rationally invariant subspace for a shift operator $S_{K}$ on a vector-valued Hardy space $H^{2}(\Omega, K)$ is generated by a quasi-inner function, we also provide relationships of quasi-inner functions by comparing rationally invariant subspaces generated by them. Furthermore, we discuss fundamental properties of quasi-inner functions and quasi-inner divisors.
\end{abstract}

\section{Introduction}

Beurling characterized all invariant subspaces for the shift operator on the Hardy space $H^{2}$ in terms of inner functions [2]. If $\varphi$ and $\phi$ are inner functions such that $\varphi H^{2} \subset \phi H^{2}$, then we have $\varphi$ is divisible by $\phi$ [3]. In fact, the converse is also true [1].

In this paper, $\Omega$ denotes a bounded finitely connected region in the complex plane and $R(\Omega)$ denotes the algebra of rational functions with poles off $\bar{\Omega}$.

For a Hilbert space $K$ and a shift operator $S_{K}$ on a vector-valued Hardy space $H^{2}(\Omega, K)$, every $R(\Omega)$-invariant (rationally invariant) subspace $M$ for the operator $S_{K}$ is characterized in terms of quasi-inner functions [4]; $M=$ $\psi H^{2}\left(\Omega, K^{\prime}\right)$ for some quasi-inner function $\psi: \Omega \rightarrow L\left(K^{\prime}, K\right)$ and a Hilbert space $K^{\prime}$. Even though a quasi-inner function is defined as an operator-valued function in [4], by the Riesz representation theorem, we also provide a definition of a scalar-valued quasi-inner function.

For quasi-inner functions $\varphi \in H^{\infty}\left(\Omega, L\left(\mathbb{C}^{n}\right)\right)$ and $u \in H^{\infty}(\Omega)$, we discuss some relationships between operator-valued and scalar-valued quasi-inner functions (Theorem 3.4). In addition, by using a multiplication operator on a vector-valued Hardy space, we study quasi-inner functions (Corollary 3.6).

For quasi-inner functions $\theta \in H^{\infty}(\Omega)$ and $\varphi \in H^{\infty}(\Omega, K)$, we provide definitions of the following two cases;

(1) $\theta$ is divisible by $\varphi$.

Received October 14, 2008.

2000 Mathematics Subject Classification. 47A15, 47A56, 47B37, 47B38.

Key words and phrases. a generalized Beurling's theorem, Hardy spaces, quasi-inner functions, rationally invariant subspaces. 
(2) $\varphi$ is divisible by $\theta$.

With these definitions, we characterize those divisibilities by comparing $R(\Omega)$-invariant subspaces, $\theta H^{2}(\Omega, K)$ and $\varphi H^{2}(\Omega, K)$ (Theorem 4.3 and Theorem 4.4); for any quasi-inner functions $\theta \in H^{\infty}(\Omega)$ and $\varphi \in H^{\infty}(\Omega, L(K))$, the following assertions are equivalent:
(a) $\theta \mid \varphi$.
(b) $\varphi H^{\infty}(\Omega, K) \subset \theta H^{\infty}(\Omega, K)$.
(c) $\varphi H^{2}(\Omega, K) \subset \theta H^{2}(\Omega, K)$.
(d) There is a $\lambda>0$ such that $\varphi(z) \varphi(z)^{*} \leq \lambda^{2}|\theta(z)|^{2} I_{K}$ for any $z \in \Omega$.

\section{Preliminaries and notation}

In this paper, $\mathbb{C}, \bar{M}$, and $L(H)$ denote the set of complex numbers, the (norm) closure of a set $M$, and the set of bounded linear operators from $H$ to $H$ where $H$ is a Hilbert space, respectively.

\subsection{Inner functions}

Let $\mathbf{D}$ be the open unit disc. We denote by $H^{\infty}$ the Banach space of all bounded analytic functions $\phi: \mathbf{D} \rightarrow \mathbb{C}$ with the norm $\|\phi\|_{\infty}=\sup \{|\phi(z)|: z \in$ D\}.

Let $\theta$ and $\theta^{\prime}$ be two functions in $H^{\infty}$. We say that $\theta$ divides $\theta^{\prime}\left(\right.$ or $\left.\theta \mid \theta^{\prime}\right)$ if $\theta^{\prime}$ can be written as $\theta^{\prime}=\theta \cdot \phi$ for some $\phi \in H^{\infty}$. We will use the notation $\theta \equiv \theta^{\prime}$ if $\theta \mid \theta^{\prime}$ and $\theta^{\prime} \mid \theta$.

Recall that a function $u \in H^{\infty}$ is inner if $\left|u\left(e^{i t}\right)\right|=1$ almost everywhere on $\partial$ D. By Beurling's theorem on invariant subspaces of the Hardy spaces, for any inner function $\theta \in H^{\infty}$, we have that $\theta H^{2}$ is an invariant subspace for the shift operator $S: H^{2} \rightarrow H^{2}$ defined by $(S f)(z)=z f(z)$ for $f \in H^{2}$.

\subsection{Hardy spaces}

We refer to [5] for basic facts about Hardy space, and recall here the basic definitions. Let $\Omega$ be a bounded finitely connected region in the complex plane.

Definition 2.1. The space $H^{2}(\Omega)$ is defined to be the space of analytic functions $f$ on $\Omega$ such that the subharmonic function $|f|^{2}$ has a harmonic majorant on $\Omega$. For a fixed $z_{0} \in \Omega$, there is a norm on $H^{2}(\Omega)$ defined by

$$
\|f\|=\inf \left\{u\left(z_{0}\right)^{1 / 2}: u \text { is a harmonic majorant of }|f|^{2}\right\} .
$$

Let $m$ be the harmonic measure for the point $z_{0}$, let $L^{2}(\partial \Omega)$ be the $L^{2}$-space of complex valued functions on the boundary of $\Omega$ defined with respect to $m$, and let $H^{2}(\partial \Omega)$ be the set of functions $f$ in $L^{2}(\partial \Omega)$ such that $\int_{\partial \Omega} f(z) g(z) d z$ $=0$ for every $g$ that is analytic in a neighborhood of the closure of $\Omega$. If $f$ is in $H^{2}(\Omega)$, then there is a function $f^{*}$ in $H^{2}(\partial \Omega)$ such that $f(z)$ approaches $f^{*}\left(\lambda_{0}\right)$ as $z$ approaches $\lambda_{0}$ nontangentially, for almost every $\lambda_{0}$ relative to $m$. The map $f \rightarrow f^{*}$ is an isometry from $H^{2}(\Omega)$ onto $H^{2}(\partial \Omega)$. 
A function $f$ defined on $\Omega$ is in $H^{\infty}(\Omega)$ if it is holomorphic and bounded. Then, $H^{\infty}(\Omega)$ is a closed subspace of $L^{\infty}(\Omega)$ and it is a Banach algebra if endowed with the supremum norm. Finally, the mapping $f \rightarrow f^{*}$ is an isometry of $H^{\infty}(\Omega)$ onto a week*-closed subalgebra of $L^{\infty}(\partial \Omega)$.

Definition 2.2. If $K$ is a Hilbert space, then $H^{2}(\Omega, K)$ is defined to be the space of analytic functions $f: \Omega \rightarrow K$ such that the subharmonic function $\|f\|^{2}$ is majorized by a harmonic function $\nu$. Fix a point $z_{0}$ in $\Omega$ and define a norm on $H^{2}(\Omega, K)$ by

$$
\|f\|=\inf \left\{\nu\left(z_{0}\right)^{1 / 2}: \nu \text { is a harmonic majorant of }\|f\|^{2}\right\} \text {. }
$$

We will work on this vector-valued Hardy space $H^{2}(\Omega, K)$. Define a shift operator $S_{K}: H^{2}(\Omega, K) \rightarrow H^{2}(\Omega, K)$ by

$$
\left(S_{K} f\right)(z)=z f(z) .
$$

\section{Quasi-inner functions}

Let $R(\Omega)$ denote the algebra of rational functions with poles off $\bar{\Omega}$, and $T$ be an operator in $L(H)$ such that $\sigma(T) \subset \bar{\Omega}$. Then a closed subspace $M$ is said to be $R(\Omega)$-invariant (rationally invariant) for the operator $T$, if it is invariant under $u(T)$ for any function $u \in R(\Omega)$.

To characterize every $R(\Omega)$-invariant subspace for the shift operator $S_{K}$, quasi-inner function was defined in [4].

Definition 3.1. Let $K$ and $K^{\prime}$ be Hilbert spaces and let $H^{\infty}\left(\Omega, L\left(K, K^{\prime}\right)\right)$ be the Banach space of all analytic functions $\Phi: \Omega \rightarrow L\left(K, K^{\prime}\right)$ with the supremum norm. For $\varphi \in H^{\infty}\left(\Omega, L\left(K, K^{\prime}\right)\right)$, we will say that $\varphi$ is quasi-inner if there exists a constant $c>0$ such that

$$
\|\varphi(z) k\| \geq c\|k\|
$$

for every $k \in K$ and almost every $z \in \partial \Omega$.

Even though a quasi-inner function is defined as an operator-valued function, by the Riesz representation theorem, we can identify $L(\mathbb{C})$ with $\mathbb{C}$. Thus we have the following definition of a scalar-valued quasi-inner function:

Definition 3.2. For $\theta \in H^{\infty}(\Omega)$, we will say that $\theta$ is quasi-inner if there exists a constant $c>0$ such that

$$
|\theta(z)| \geq c
$$

for almost every $z \in \partial \Omega$.

Proposition 3.3. Let $K$ and $K^{\prime}$ be Hilbert spaces with $\operatorname{dim} K=\operatorname{dim} K^{\prime}=$ $n(<\infty)$.

If $\varphi \in H^{\infty}\left(\Omega, L\left(K, K^{\prime}\right)\right)$ is a quasi-inner function, then $\varphi(z)$ is invertible a.e. on $\partial \Omega$. 
Proof. Since $\varphi \in H^{\infty}\left(\Omega, L\left(K, K^{\prime}\right)\right)$ is quasi-inner, there is a set $A \subset \partial \Omega$ with $m(A)=0$ such that the range of $\varphi\left(z_{0}\right)$ is closed, and $\varphi\left(z_{0}\right)$ is one-to-one for any $z_{0} \in \partial \Omega \backslash A$.

Thus, $K$ and the range of $\varphi\left(z_{0}\right)$ have the same dimension.

Since $\operatorname{dim} K=\operatorname{dim} K^{\prime}$, we conclude that the range of $\varphi\left(z_{0}\right)$ is $K^{\prime}$. Thus $\varphi(z)$ is invertible for $z \in \partial \Omega \backslash A$.

Theorem 3.4. (a) If $\varphi \in H^{\infty}\left(\Omega, L\left(\mathbb{C}^{n}\right)\right)$ and $u \in H^{\infty}(\Omega)$ are quasi-inner functions such that

$$
\varphi(z) \psi(z)=u(z) I_{\mathbb{C}^{n}}
$$

where $\psi \in H^{\infty}\left(\Omega, L\left(\mathbb{C}^{n}\right)\right)$, then $\psi$ is also quasi-inner.

(b) Conversely, if $\varphi \in H^{\infty}\left(\Omega, L\left(\mathbb{C}^{n}\right)\right)$ and $\psi \in H^{\infty}\left(\Omega, L\left(\mathbb{C}^{n}\right)\right)$ are quasiinner functions such that

$$
\varphi(z) \psi(z)=u(z) I_{\mathbb{C}^{n}} \quad \text { or } \quad \psi(z) \varphi(z)=u(z) I_{\mathbb{C}^{n}}
$$

for some $u \in H^{\infty}(\Omega)(u \neq 0)$, then $u$ is quasi-inner.

Proof. (a) Since $\varphi$ and $u$ are quasi-inner functions, there are constants $m_{1}(>0)$ and $c_{i}(>0)(i=1,2)$ such that

(i) $m_{1} \leq|u(z)|$ a.e. on $\partial \Omega$, and

(ii) for $h \in \mathbb{C}^{n}, c_{1}\|h\| \leq\|\varphi(z) h\| \leq c_{2}\|h\|$ a.e. on $\partial \Omega$.

Since $\varphi(z) \psi(z)=u(z) I_{\mathbb{C}^{n}}$ for $h \in \mathbb{C}^{n}, m_{1}\|h\| \leq|u(z)|\|h\|=\|\varphi(z) \psi(z) h\| \leq$ $c_{2}\|\psi(z) h\|$ a.e. on $\partial \Omega$. Thus, for $h \in \mathbb{C}^{n}$,

$$
\frac{m_{1}}{c_{2}}\|h\| \leq\|\psi(z) h\|
$$

a.e. on $\partial \Omega$.

From (3.1), we conclude that $\psi$ is also quasi-inner.

(b) Since $\varphi \in H^{\infty}\left(\Omega, L\left(\mathbb{C}^{n}\right)\right)$ and $\psi \in H^{\infty}\left(\Omega, L\left(\mathbb{C}^{n}\right)\right)$ are quasi-inner functions, there exist $m_{1}(>0)$ and $m_{2}(>0)$ such that, for $h \in \mathbb{C}^{n}, m_{1}\|h\| \leq$ $\|\varphi(z) h\|$ a.e. on $\partial \Omega$ and $m_{2}\|h\| \leq\|\psi(z) h\|$ a.e. on $\partial \Omega$. Then

$$
\|\varphi(z) \psi(z) h\| \geq m_{1}\|\psi(z) h\| \geq m_{1} m_{2}\|h\|
$$

and so $\|\varphi(z) \psi(z)\| \geq m_{1} m_{2}$ a.e. on $\partial \Omega$. Since $|u(z)|=\|\varphi(z) \psi(z)\|$, it is proven.

Furthermore, by using these quasi-inner functions, we have a generalization of Beurling's theorem as following:

Theorem A (Theorem 1.5 in [4]). Let $K$ be a Hilbert space. Then a closed subspace $M$ of $H^{2}(\Omega, K)$ is $R(\Omega)$-invariant for $S_{K}$ if and only if there is a Hilbert space $K^{\prime}$ and a quasi-inner function $\varphi: \Omega \rightarrow L\left(K^{\prime}, K\right)$ such that $M=$ $\varphi H^{2}\left(\Omega, K^{\prime}\right)$.

Since we have two kinds of quasi-inner functions, we have two kinds of $R(\Omega)$-invariant subspaces for $S_{K}$. One of them is generated by a scalar-valued quasi-inner function, and the other one is generated by an operator-valued 
quasi-inner function. We will also compare these two $R(\Omega)$-invariant subspaces for $S_{K}$ in Theorem 4.3.

Let $K_{1}$ and $K_{2}$ be separable Hilbert spaces. To discuss quasi-inner functions, we define a multiplication operator for a given function $\psi \in H^{\infty}\left(\Omega, L\left(K_{1}, K_{2}\right)\right)$. A multiplication operator $M_{\psi}: H^{2}\left(\Omega, K_{1}\right) \rightarrow H^{2}\left(\Omega, K_{2}\right)$ is defined by

$$
M_{\psi}(g)(z)=\psi(z) g(z)
$$

for all $g$ in $H^{2}\left(\Omega, K_{1}\right)$. We can easily check that $\left\|M_{\psi}\right\|=\|\psi\|_{\infty}$.

Recall an important property of this multiplication operator:

Proposition 3.5 ([4]). Let $K_{1}$ and $K_{2}$ be separable Hilbert spaces. If $T$ : $H^{2}\left(\Omega, K_{1}\right) \rightarrow H^{2}\left(\Omega, K_{2}\right)$ is a bounded linear operator such that $T S_{K_{1}}=S_{K_{2}} T$, then there is a function $\psi \in H^{\infty}\left(\Omega, L\left(K_{1}, K_{2}\right)\right)$ such that $T=M_{\psi}$.

Theorem 3.6. Let $\varphi \in H^{\infty}\left(\Omega, L\left(K_{1}, K_{2}\right)\right)$.

(a) If $\varphi$ is quasi-inner, then $M_{\varphi}$ is one-to-one and has closed range.

(b) If $M_{\varphi}: H^{2}\left(\Omega, K_{1}\right) \rightarrow H^{2}\left(\Omega, K_{2}\right)$ is invertible, then $\varphi$ is quasi-inner.

Proof. (a) By Theorem A, $M_{\varphi} H^{2}\left(\Omega, K_{1}\right)=\varphi H^{2}\left(\Omega, K_{1}\right)$ is closed.

Since $\varphi(z)$ is a bounded below operator a.e. on $\partial \Omega, f \in \operatorname{ker} M_{\varphi}=\{f \in$ $\left.H^{2}\left(\Omega, K_{1}\right): \varphi(z) f(z)=0(z \in \Omega)\right\}$ if and only if $f^{*} \equiv 0$ in $H^{2}\left(\partial \Omega, K_{1}\right)$ if and only if $f \equiv 0$.

(b) Since $\varphi H^{2}\left(\Omega, K_{1}\right)$ is $R(\Omega)$-invariant for $S_{K_{2}}$, by Theorem A,

$$
\varphi H^{2}\left(\Omega, K_{1}\right)=\varphi_{1} H^{2}\left(\Omega, K_{0}\right)
$$

for a Hilbert space $K_{0}$ and a quasi-inner function $\varphi_{1}: \Omega \rightarrow L\left(K_{0}, K_{2}\right)$.

Define a linear operator $T: H^{2}\left(\Omega, K_{1}\right) \rightarrow H^{2}\left(\Omega, K_{0}\right)$ as follows. For $f \in H^{2}\left(\Omega, K_{1}\right), T f=g$ such that $\varphi f=\varphi_{1} g$. Since $\varphi_{1}$ is a quasi-inner function, by (a), $T$ is well-defined and $T$ is bounded. Since $S_{K_{0}} T=T S_{K_{1}}$, by Proposition 3.5, $T=M_{\varphi_{2}}$ for a function $\varphi_{2} \in H^{\infty}\left(\Omega, L\left(K_{1}, K_{2}\right)\right)$. It follows that

$$
\varphi(z)=\varphi_{1}(z) \varphi_{2}(z)
$$

for any $z \in \Omega$.

Since $M_{\varphi}$ is onto, so is $M_{\varphi_{1}}$. By (a), $M_{\varphi_{1}}$ is one-to-one, and so $M_{\varphi_{1}}$ is invertible. Since $M_{\varphi}$ and $M_{\varphi_{1}}$ are invertible, so is $T=M_{\varphi_{2}}$. Note that the invertibility of $M_{\varphi}$ is equivalent to the invertibility of $\varphi(z)$ for any $z$ in $\Omega$. It follows that $\varphi_{2}(z)$ is bounded below for any $z$ in $\Omega$.

Since $\varphi_{1}$ is quasi-inner, $\varphi_{1}(z)$ is also bounded below a.e. on $\partial \Omega$.

Therefore, by equation (3.2), for any $a \in K_{1}$, there is a constant $c>0$ such that

$$
\|\varphi(z) a\| \geq c\|a\|
$$

a.e. on $\partial \Omega$. 


\section{Quasi-inner divisors}

Let $K$ be a Hilbert space. The time has come to consider divisibilities between a function in $H^{\infty}(\Omega)$ and a function in $H^{\infty}(\Omega, L(K))$.

Definition 4.1. If $\theta \in H^{\infty}(\Omega)$ and $\varphi \in H^{\infty}(\Omega, L(K))$, then we say that $\theta$ divides $\varphi$ (denoted $\theta \mid \varphi$ ) if $\varphi$ can be written as

$$
\varphi=\theta \cdot \phi^{\prime}
$$

for some $\phi^{\prime} \in H^{\infty}(\Omega, L(K))$.

Definition 4.2. If $\theta \in H^{\infty}(\Omega)$ and $\varphi \in H^{\infty}(\Omega, L(K))$, then we say that $\varphi$ divides $\theta$ (denoted $\varphi \mid \theta$ ) if there exists $\psi \in H^{\infty}(\Omega, L(K)$ ) satisfying the following relations;

$$
\varphi(z) \psi(z)=\theta(z) I_{K}
$$

and

for $z \in \Omega$.

$$
\psi(z) \varphi(z)=\theta(z) I_{K}
$$

Theorem 4.3. For any quasi-inner functions $\theta \in H^{\infty}(\Omega)$ and $\varphi \in H^{\infty}(\Omega, L(K))$, the following assertions are equivalent:
(a) $\theta \mid \varphi$
(b) $\varphi H^{\infty}(\Omega, K) \subset \theta H^{\infty}(\Omega, K)$.
(c) $\varphi H^{2}(\Omega, K) \subset \theta H^{2}(\Omega, K)$.
(d) There is a $\lambda>0$ such that $\varphi(z) \varphi(z)^{*} \leq \lambda^{2}|\theta(z)|^{2} I_{K}$ for any $z \in \Omega$, where $I_{K}$ is the identity function on $K$.

Proof. If $\theta \mid \varphi, \varphi=\theta \varphi_{1}$ for some $\varphi_{1} \in H^{\infty}(\Omega, L(K))$. Then

$$
\varphi H^{\infty}(\Omega, K)=\theta \varphi_{1} H^{\infty}(\Omega, K) \subset \theta H^{\infty}(\Omega, K) .
$$

Thus (a) implies (b).

Conversely, suppose that $\varphi H^{\infty}(\Omega, K) \subset \theta H^{\infty}(\Omega, K)$. Then

$$
\varphi^{*} H^{\infty}(\partial \Omega, K) \subset \theta^{*} H^{\infty}(\partial \Omega, K) .
$$

Let $\left\{b_{i}: i \in I\right\}$ be an orthonormal basis of $K$ and $g_{i} \in H^{\infty}(\partial \Omega, K)$ defined by $g_{i}(z)=b_{i}(i \in I)$. By (4.1), there is $f_{i} \in H^{\infty}(\partial \Omega, K)$ such that $\varphi^{*} g_{i}=\theta^{*} f_{i}$, i.e., for $i \in I$,

$$
\varphi^{*}(z) b_{i}=\theta^{*}(z) f_{i}(z) .
$$

Define $\varphi_{1}: \partial \Omega \rightarrow L(K)$ by for $i \in I$,

$$
\varphi_{1}(z) b_{i}=f_{i}(z) .
$$

For $i \in I$, define $\varphi_{i} \in H^{\infty}(\partial \Omega, L(K))$ by $\varphi_{i}(z) b_{j}=\delta_{i j} f_{i}(z)(j \in I)$, where $\delta_{i j}=\left\{\begin{array}{ll}1 & \text { if } i=j \\ 0 & \text { otherwise. }\end{array}\right.$ Then

$$
\varphi_{1}=\sum_{i \in I} \varphi_{i}
$$


By (4.2) and (4.3), for each $i \in I, \varphi^{*}(z) b_{i}=\theta^{*}(z) \varphi_{1}(z) b_{i}$, and so

$$
\varphi^{*}=\theta^{*} \varphi_{1}
$$

To prove that (b) implies (a), we have to show that $\varphi_{1} \in H^{\infty}(\partial \Omega, L(K))$. Since $\theta \in H^{\infty}$ is a quasi-inner function, there is $c>0$ such that $|\theta(z)| \geq c$ for every $z \in A \subset \partial \Omega$ with $m(\partial \Omega \backslash A)=0$. For any $x \in K$ with $\|x\|=1$ and $z \in A$,

$$
\left\|\varphi_{1}(z) x\right\|=\frac{\left\|\varphi^{*}(z) x\right\|}{\left|\theta^{*}(z)\right|} \leq \frac{\|\varphi\|_{\infty}}{c} .
$$

From (4.4) and (4.5), we conclude that

$$
\varphi_{1} \in H^{\infty}(\partial \Omega, L(K)) .
$$

By the same way as above, $(\mathrm{a}) \Leftrightarrow(\mathrm{c})$ is proven. We begin to prove $(\mathrm{a}) \Leftrightarrow(\mathrm{d})$. If $\theta \mid \varphi, \varphi=\theta \varphi_{1}$ for some $\varphi_{1} \in H^{\infty}(\Omega, L(K))$. Then

$$
\varphi(z) \varphi(z)^{*}=\theta(z) \varphi_{1}(z) \varphi_{1}(z)^{*} \overline{\theta(z)} \leq\left\|\varphi_{1}\right\|_{\infty}^{2}|\theta(z)|^{2} I_{K} .
$$

Let $\lambda=\left\|\varphi_{1}\right\|_{\infty}$. Since $\varphi$ is quasi-inner, $\varphi \neq 0$ and so $\lambda>0$. Thus (a) implies (d).

Conversely, suppose that for any $z \in \Omega$,

$$
\varphi(z) \varphi(z)^{*} \leq \lambda^{2}|\theta(z)|^{2} I_{K}
$$

for some $\lambda>0$. For each $z \in \Omega$, we will define a linear mapping $F_{z} \in L(K)$. Let

and

$$
A=\{z \in \Omega: \theta(z)=0\}
$$

$$
B=\{z \in \Omega: \theta(z) \neq 0\} .
$$

If $z \in A$, then let $F_{z}=0$. If $z \in B$, then range of $\overline{\theta(z)} I_{K}$ is $K$ and so we can define a linear mapping $F_{z}$ from $K$ to range of $\varphi(z)^{*}$ by

$$
F_{z}(\overline{\theta(z)} f)=\varphi(z)^{*} f
$$

for $f \in K$.

Since $\left\|F_{z}(\overline{\theta(z)} f)\right\|^{2}=\left\|\varphi(z)^{*} f\right\|^{2}=\left(\varphi(z) \varphi(z)^{*} f, f\right) \leq \lambda^{2}\left(|\theta(z)|^{2} f, f\right)=$ $\lambda^{2}\|\theta(z) f\|^{2}$, that is,

$$
\left\|F_{z}(\overline{\theta(z)} f)\right\| \leq \lambda\|\theta(z) f\|,
$$

$F_{z}$ is well-defined for $z \in B$. By definition of $F_{z}$, if $z \in B$,

$$
\theta(z) F_{z}^{*}=\varphi(z) \text {. }
$$

If $z \in A$, by (4.6) $\|\varphi(z)\|=0$ and so $\varphi(z)=0(z \in A)$. Thus $\theta(z) F_{z}^{*}=\varphi(z)$ for any $z \in \Omega$.

Define a function $F: \Omega \rightarrow L(K)$ by

$$
F(z)=F_{z}^{*} .
$$


Then by equation (4.8),

$$
\varphi(z)=\theta(z) F(z)
$$

for $z \in \Omega$. To finish this proof, we have to prove that $F \in H^{\infty}(\Omega, L(K))$. From inequality (4.7), we have

$$
\|F\|_{\infty} \leq \lambda
$$

and so $F=\frac{\varphi}{\theta}$ has only removable singularities in $\Omega$. Thus $F$ can be defined on $\{z \in \Omega: \theta(z)=0\}$ so that $F$ is analytic and

$$
\varphi=\theta F \text {. }
$$

From (4.9), $F \in H^{\infty}(\Omega, L(K))$ which proves $(\mathrm{d}) \Rightarrow(\mathrm{a})$.

We have another result similar to Theorem 4.3.

Theorem 4.4. For any quasi-inner functions $\theta \in H^{\infty}(\Omega)$ and $\varphi \in H^{\infty}(\Omega, L(K))$, the following assertions are equivalent:
(a) $\varphi \mid \theta$.
(b) $\theta H^{\infty}(\Omega, K) \subset \varphi H^{\infty}(\Omega, K)$.
(c) $\theta H^{2}(\Omega, K) \subset \varphi H^{2}(\Omega, K)$.
(d) There is a $\lambda>0$ such that $|\theta(z)|^{2} I_{K} \leq \lambda^{2} \varphi(z) \varphi(z)^{*}$ for any $z \in \Omega$.

Proof. This theorem is proven by the same way as Theorem 4.3.

\section{References}

[1] H. Bercovici, Operator Theory and Arithmetic in $H^{\infty}$, Mathematical Surveys and Monographs, 26. American Mathematical Society, Providence, RI, 1988.

[2] A. Beurling, On two problems concerning linear transformations in Hilbert space, Acta Math. 81 (1948), 17 pp.

[3] Paul R. Halmos, A Hilbert Space Problem Book, van Nostrand-Reinhold, Princeton, New Jersey, 1967.

[4] Y. S. Kim, Linear algebraic properties for Jordan models of $C_{0}$-operators relative to multiply connected domains, J. Operator Theory 57 (2007), no. 2, 375-389.

[5] W. Rudin, Analytic functions of class $H_{p}$, Trans. Amer. Math. Soc. 78 (1955), 46-66.

[6] B. Sz.-Nagy and C. Foias, Harmonic Analysis of Operators on Hilbert Space, NorthHolland, Amsterdam, 1970.

[7] A. Zucchi, Operators of class $C_{0}$ with spectra in multiply connected regions, Mem. Amer. Math. Soc. 127 (1997), no. 607, viii+52 pp.

Department of Mathematics

The University of Toledo

2801 W. Bancroft St. Toledo, OH 43606, U. S. A.

E-mail address: Yun-Su.Kim@utoledo.edu 